include obtaining samples for toxicology, biopsy of lesions, minimally invasive vascular access, and contrast enhanced imaging studies.

\section{THE FORMATION OF A CARDIAC ARREST REGISTRY IN AUSTRALIA [END UNEXPLAINED CARDIAC DEATH (EndUCP) REGISTRY]}

\section{Sarah Parsons ${ }^{1,2}$, Elizabeth D. Paratz ${ }^{3,4,5}$,}

Alexander van Heusden ${ }^{3}$, Dominica Zentner ${ }^{6,7}$, Natalie Morgan ${ }^{1}$, Tina Thompson ${ }^{6}$, Paul James ${ }^{6}$,

Andreas Pflaumer ${ }^{8,9,10}$, Christopher Semsarian ${ }^{11}$, Jodie Ingles $^{12,13,14}$, Rosalind Case ${ }^{3,4,15,16,17}$, Jocasta Ball ${ }^{3,16,18}$, Karen Smith ${ }^{16,18}$, Dion Stub ${ }^{3,4,15,16}$,

Andre La Gerche ${ }^{3,4,5}$, for the Australian EndUCD Registry

${ }^{1}$ Victorian Institute of Forensic Medicine, Melbourne, Vic,

Australia; ${ }^{2}$ Department of Forensic Medicine, Monash University, Melbourne, Vic, Australia; ${ }^{3}$ Baker Heart and Diabetes Institute, Melbourne, Vic, Australia; ${ }^{4}$ Alfred Hospital, Melbourne, Vic, Australia; ${ }^{5}$ St Vincent's Hospital Melbourne, Melbourne, Vic, Australia; ${ }^{6}$ Royal Melbourne Hospital, Melbourne, Vic, Australia; ${ }^{7}$ Royal Melbourne Hospital Clinical School, Faculty of Medicine, Dentistry and Health Sciences, University of Melbourne, Melbourne, Vic, Australia; ${ }^{8}$ Royal Children's Hospital, Melbourne, Vic, Australia; ${ }^{9}$ Department of Paediatrics, Melbourne University, Melbourne, Vic, Australia; ${ }^{10}$ Murdoch Children's Research Institute, Royal Children's Hospital, Melbourne, Vic, Australia; ${ }^{11}$ Agnes Ginges Centre for Molecular Cardiology at Centenary Institute, The University of Sydney, Sydney, NSW, Australia; ${ }^{12}$ Cardio Genomics Program at Centenary Institute, The University of Sydney, Sydney, NSW, Australia; ${ }^{13}$ Department of Cardiology, Royal Prince Alfred Hospital, Sydney, NSW, Australia; ${ }^{14}$ Faculty of Medicine and Health, The University of Sydney, Sydney, NSW, Australia;

${ }^{15}$ Department of Epidemiology and Preventive Medicine, Monash University, Melbourne, Vic, Australia; ${ }^{16}$ Ambulance Victoria, Melbourne, Vic, Australia; ${ }^{17}$ Florey Institute of Neuroscience and Mental Health, Melbourne, Vic, Australia; and ${ }^{18}$ Department of Community Emergency Health and Paramedic Practice, Monash University, Melbourne, Vic, Australia

The End Unexplained Cardiac Death (EndUCD) Registry commenced in Victoria, Australia in April 2019. It is a multicentre cardiac arrest surveillance registry for patients aged 1 to 50 years. It is intended to expand into NSW and then other states of Australia. The key aims of the EndUCD Registry are to:

1) Provide a patient support resource, including website, patientoriented information and streamlined referral process.

2) Create a research platform for advocacy and improving understanding of accurate rates of sudden cardiac arrest/ death in patients aged $1-50$ years old.

3) Create a genetic biorepository as a resource for future studies.

4) Provide a state-of-the-art resource for clinicians wishing to link cardiac arrest patients and families in with psychological support, comprehensive family screening and consideration of genetic testing.

This presentation will examine the initial formation of the registry and present initial data from the first 2 years of collection and examine the role of forensic pathologist and forensic services in such a registry.

\section{BACKGROUND, TESTING METHODS, AND LABORATORY APPROACHES TO SARS CORONAVIRUS-2 (SARS-CoV-2) AND COVID19}

$\underline{\text { William D. Rawlinson }}^{1,2}$, Sebastiaan van $\mathrm{Hal}^{3}$, Malinna Yeang ${ }^{1}$, Anna Condylios $^{1}$, Zin Naing ${ }^{1}$, Jane-Phan $\mathrm{Au}^{1,2}$, Mariana Ruiz da Silva ${ }^{1,2}$, Charles Foster ${ }^{1,2}$

${ }^{1}$ Serology and Virology Division, NSW Health Pathology Microbiology, SEALS Prince of Wales Hospital, NSW, Australia; ${ }^{2}$ Schools of Women's and Children's Health, Medicine and Biotechnology and Biomolecular Sciences, University of New South Wales, Sydney, NSW, Australia; and ${ }^{3}$ Department of Microbiology and Infectious Diseases, NSW Health Pathology, Royal Prince Alfred Hospital, NSW, Australia

Introduction: A pandemic is an epidemic occurring worldwide, crossing international boundaries and affecting many people. The spread of SARS-CoV-2 from Hubei province in China globally has led to rapid changes in our approaches to diagnosis, with rapid use of molecular methods such as whole genome sequencing (WGS) to characterise mutations, and deployment of novel therapeutics including mRNA vaccines and monoclonal antibodies.

Methods: New methods for diagnosis, including WGS, and different approaches to emerging mutations in virus variants of concern (VOC) will be discussed.

Results and conclusions: Diagnosis now uses standard molecular methods including commercial and inhouse techniques, more recently rapid antigen detection, and research into rapid smartphone based nanoparticle assays. Laboratory practices of sample pooling, reflex testing using WGS, and workflow changes are now routine.

The use of rapid WGS techniques, ${ }^{1}$ and available Australian data, ${ }^{2,3}$ have informed assessment of the accuracy and utility of these methods in the diagnostic laboratory. Assessment of changes in PCR primer binding sites, and of the influence of how sequence data are assessed (Foster et al., in press) means accreditation and QA programs need to be continuously updated. References

1. Bull RA, Adikari TN, Ferguson JM, et al. Analytical validity of nanopore sequencing for rapid SARS-CoV-2 genome analysis. Nat Commun 2020; 11: 6272.

2. Rockett RJ, Arnott A, Lam C, et al. Revealing COVID-19 transmission in Australia by SARS-CoV-2 genome sequencing and agent-based modeling. Nat Med 2020; 26: 1398-404.

3. Seemann T, Lane CR, Sherry NL, et al. Tracking the COVID19 pandemic in Australia using genomics. Nat Commun 2020; 11: 4376 .

\section{CORONIAL CASES: SARS-CoV-2 INFECTION AND COVID-19}

Linda Iles

Victorian Institute of Forensic Medicine, Melbourne, Vic, Australia

Victoria's second wave of SARS-CoV-2 infections saw a number of reports of deaths of SARS-CoV-2 positive persons made to the coroner. Whilst COVID-19 deaths are natural and do not routinely fall under the coroner's jurisdiction, these cases were brought to the coroner's attention due to deaths 